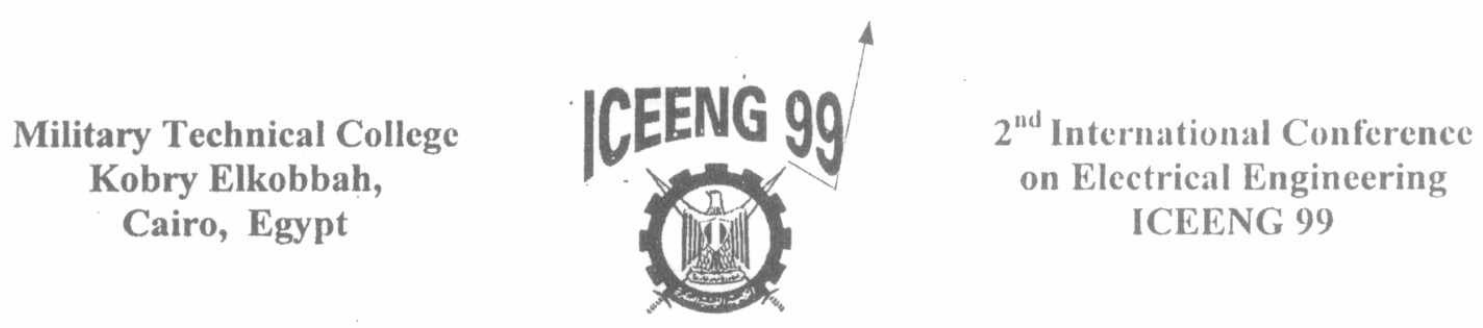

\title{
ON THE PERFORMANCE ANALYSIS AND SPECIFICATION OF A RADAR PROXIMITY FUSE
}

\author{
A. H. ASSEESY ${ }^{1}$
}

\begin{abstract}
In this paper the main aspects, system specification, architecture and design of radar proximity fuses are discussed. Complete analytical expressions have been developed by the author and numerical results have been achieved in typical cases. Different principles for radar proximity fuses are analyzed and compared and a hybrid fuse architecture has been proposed. The most important design criteria for the proposed fuse have been discussed and analyzed. Calculated results have been introduced in tabular and graphical forms.
\end{abstract}

\section{KEY WORDS}

radar proximity fuse, Doppler fuse, energy fuse, height resolution, hybrid fuse, chopping, duty ratio, chopping rate, accumulated energy.

\section{NOMENCLATURE}

$h_{\max }, h_{\min }, f_{[M]}, \Delta f_{d}, P_{T}, P_{R}, \alpha_{0}, \sigma_{G}, \sigma_{0}, \theta_{\max }, T, \delta_{R}$

\section{INTRODUCTION}

Fuse system design requirements depend cn the ertillery shell caliber, launch angle and initial speed. They depend also on the background radar crossection at the target area. However, the most important common design requirement is its height resolution; specially at low altitudes. Another requirement is signal selectivity; from which interference and jamming immunity are logical developments. A successful fuse detonates at a relatively low altitude of the order of 10 meters; in order to give the most lethal destruction capabilities. In the following sections we shall discuss different fuse architectures, analyze and compare their performances. A hybrid fuse

\footnotetext{
'Ph.D., EW Engineering Department, MTC,.
} 
ystem architecture is proposed, explained and some of its design aspects are discussed.

\section{BASIC EQUATIONS OF A SHELI TRAJECTORY}

Given an artillery shell launched at an initial angle $\alpha_{0}$ with an initial velocity $v_{0}$; assuming that the gun and the target are at the same altitude and neglecting the wind resistance to simplify the model $^{2}$; the motion in the vertical plane is affected only by the gravitation force $\mathbf{m g}$ and the following relations hold [1] :-

Since the proximity fuse will not operate in the ascending part of the shell trajectory; we are interested only in the descending pari; starting at the projectile crest where the vertical velocity component vanishes. The maximum height attained by the shell is a function of the initial angle $\alpha_{0}$ and the initial velocity $v_{0}$

$$
h_{\max }=\frac{v_{0}^{2} \sin ^{2}\left(\alpha_{0}\right)}{2 g}
$$

The vertical velocity component is a linear function of time and the height is a quadratic one:

$$
\begin{aligned}
& \dot{h}=g \cdot t \\
& h=h_{\max }-\frac{g \cdot t^{2}}{2}
\end{aligned}
$$

where $t$ is the time starting at the projectile crest.

\section{DOPPLER FUSE ANAL.YSIS}

We can rearrange the last two equations to get the vertical velocity as a function of height in the descending part of the trajectory:

$$
\begin{aligned}
& t=\sqrt{\frac{2}{g} \cdot\left(h_{\max }-h\right)} \\
& \dot{h}=\sqrt{2 g \cdot\left(h_{\max }-h\right)}
\end{aligned}
$$

The Doppler shift between the reflected wave and the transmitted one is proportional to the relative velocity between the fuse c.nd the ground surface; i.e. the time rate of height variation:

\footnotetext{
'If we consider a wind resistance $=k v^{2}$ where $v$ is the shall velocity; we get the following pair of differential equations to be solved simultaneously for $\mathrm{x}(\mathrm{t})$ and $\mathrm{y}(\mathrm{t})[1]$ :

$m \ddot{x}+k \dot{x} \sqrt{\left(\dot{x}^{2}+\dot{y}^{2}\right)}=0 \quad$ and $\quad m(g+\ddot{y})+k \dot{y} \sqrt{\left(\dot{x}^{2}+\dot{y}^{2}\right)}=0$
} 


$$
f_{d}=\frac{2 \dot{h}}{\lambda}=\frac{2 \cdot f_{[M]} \cdot \sqrt{2 g \cdot\left(h_{\max }-h\right)}}{c} \cong 0.02953 f_{[M]} \cdot \sqrt{\left(h_{\max }-h\right)}
$$

where $f_{[M]}$ is the carrier frequency $[\mathrm{MHz}]$ and $c$ is the wave propagation velocity. Differentiating (6) we get:

$$
\frac{\partial f_{d}}{\partial(h)}=\frac{-0.01477 f_{[M]}}{\sqrt{\left(h_{\max }-h\right)}} \quad[\mathrm{Hz} / \mathrm{m}]
$$
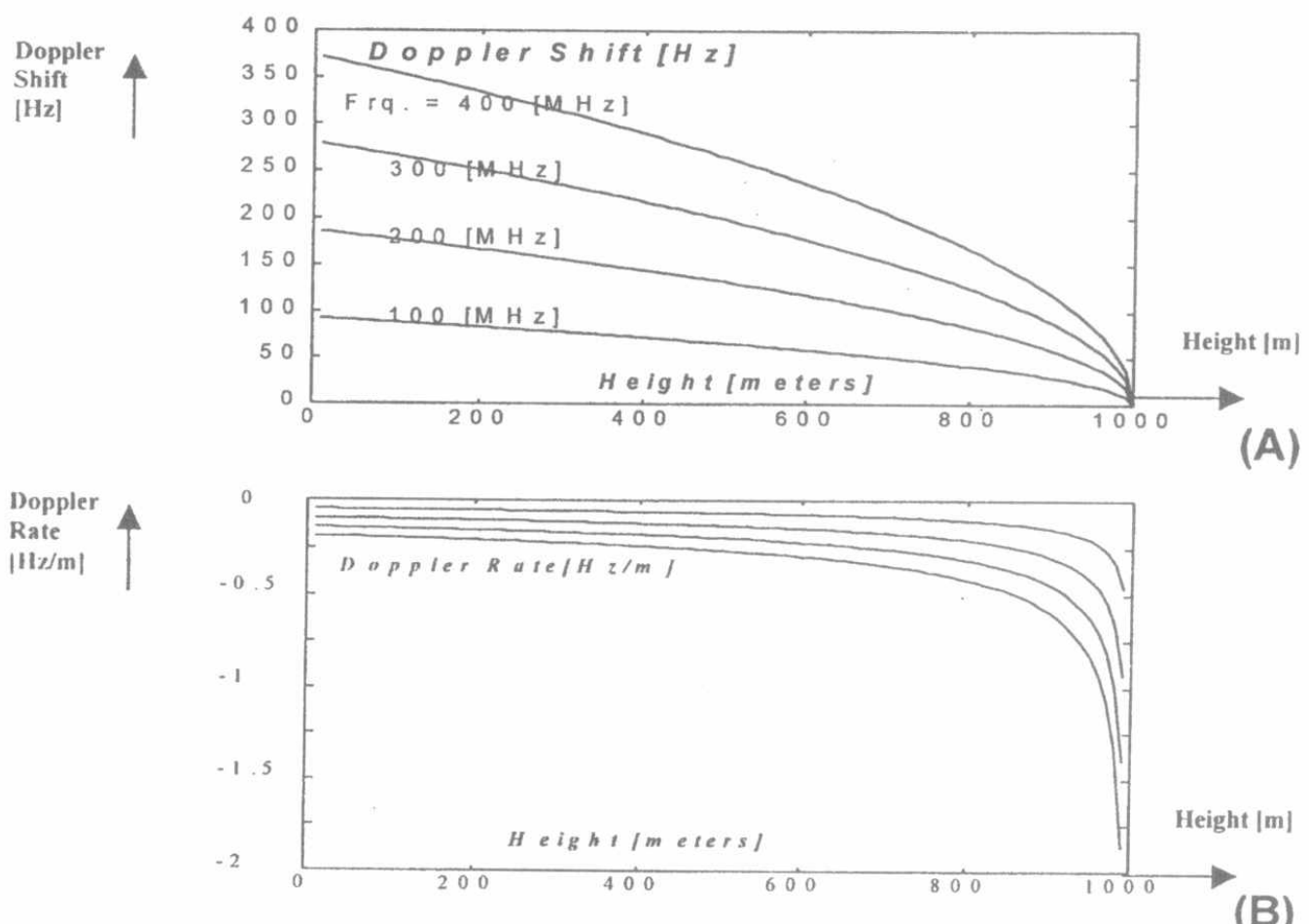

Fig.1 Variation and variation rate of doppler shift with height

Plots of (6) and (7) are shown in Fig.1 for different UHF carrier frequencies; assuming a $1[\mathrm{~km}]$ maximum height. It is evident that the negative rate of variation of $f_{d}$ with height decreases sharply in the first part of the descending trajectory and remains very small until the shell reachas the ground. The result is that the Doppler sensing system sensitivity is too low to differentiate between 200 and 10 meters of height. The difference between the measured Doppler shifts at 10 and 200 meters is given by:

$$
\Delta f_{d}=0.02953 . f_{[M]}\left[\left(\sqrt{h_{\max }-200}\right)-\left(\sqrt{h_{\max }-10}\right)\right]
$$


This difference is proportional to the carrier frequency, but it decreases considerably with the increase of the maximum height; i.e. with the increase of the projectile range. The results are given in the following table.

Table 1: Doppler Shift Differences between 200 [m] and 10 [m] Heights

\begin{tabular}{|ccc|}
\hline$h_{\max }[\mathbf{m}]$ & $\Delta \mathbf{f}_{\mathrm{d}}[\mathbf{H z}]$ & $\Delta \mathbf{f}_{\mathrm{d}}[\mathbf{H z}]$ \\
& for $\mathbf{1 0 0}[\mathbf{M} \mathbf{H z}]$ & for $\mathbf{4 0 0}[\mathbf{M H z}]$ \\
1000 & 9 & 40 \\
10000 & 3 & 12 \\
\hline
\end{tabular}

We can conclude that a Doppler fuse has a relatively low height resolution, specially for long range projectiles.

\section{ENERGY FUSE ANALYSIS}

In this fuse type the decision is based on comparing the energy (accumulated power) reflected from the ground to a certain thresho!d corresponding to its expected level at the desired height. To estimate that threshold level we proceed as follows: Let the fuse transmit a power $\boldsymbol{P}_{T}$ with a unity antenna gain. The reflected power will be:

$$
P_{r}=\frac{P_{T} \cdot \sigma_{(i} \cdot \lambda^{2}}{(4 \pi)^{3} R^{4}}
$$

where $\sigma_{G}$ is the radar crossection of the entire ground area illuminated simultaneously by the fuse transmitter $\left[\mathrm{m}^{2}\right]$

$\lambda$ is the radar wave length [ $\mathrm{m}]$

$R$ is the slant range between the radar fuse and the ground [m]

Since $R^{4}=\left(r^{2}+h^{2}\right)^{2}$ is not constant for different points within that area of the ground illuminated by the fuse transmitter; we compute the power reflected from an elementary area $(\boldsymbol{r} \boldsymbol{d} \boldsymbol{r} \boldsymbol{d} \varphi)$ and integrate over the whole illuminated area. Assuming an omni-directional antenna illuminating an open ground until the maximum line-ofsight range (given by the famous formula $r_{\max } \cong 4.11 \sqrt{h}$ ); the total reflected power is:

$$
P=\int_{0}^{2 \pi} \int_{0}^{4110 \sqrt{h}} \frac{P_{T} \cdot \lambda^{2} \cdot \sigma_{0}}{(4 \pi)^{3}\left(h^{2}+r^{2}\right)^{2}} \cdot r \cdot d r \cdot d \varphi
$$




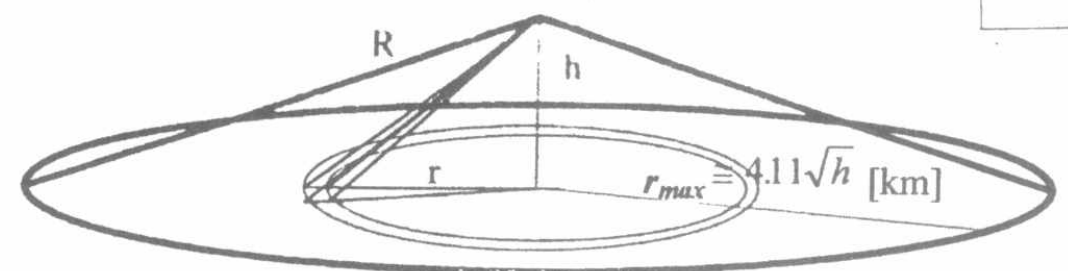

Fig.2 Computation of the radar crossection of the ground

where $\sigma_{0}$ is the radar crossection of unit area in the zone of interest (of the order of $0.01\left[\mathrm{~m}^{2} / \mathrm{m}^{2}\right]$ for concrete and $0.0001\left[\mathrm{~m}^{2} / \mathrm{m}^{2}\right]$ for sand).

Making the substitution $\Theta=2 \arctan (r / h)$; we get:

$$
P=\int_{0}^{2 \pi} \int_{0}^{\left[\Theta_{\max }\right]} \frac{P_{T} \cdot \lambda^{2} \cdot \sigma_{0}}{(4 \pi)^{3}(2 h)^{2}} \cdot \sin \Theta \cdot d \Theta \cdot d \varphi
$$

where $\Theta_{\max }=2 \tan ^{-1}(4110 / \sqrt{h})$

The total power at the fuse receiver input will be:

$$
P=\frac{P_{T} \cdot \lambda^{2} \cdot \sigma_{0}}{128 \pi^{2} h^{2}} \cdot\left(1-\cos \left(\Theta_{\max }\right)\right)
$$

Since the fuse operates in the final part of the path where $\mathrm{h}<500[\mathrm{~m}]$ and the value of $\Theta_{\max }$ is very near to $180^{\circ}$; we can approximate the formula (13) to:

$$
P \cong \frac{P_{T} \cdot \lambda^{2} \cdot \sigma_{0}}{64 \pi^{2} h^{2}}=P_{T} \cdot \sigma_{0} \cdot\left(\frac{75}{2 \pi \cdot h \cdot f_{[M]}}\right)^{2}
$$

The detonation decision is taken when $h$ reaches the desired height $\approx 10[\mathrm{~m}]$. It is evident from (14) that the power received at a 10 [m] is $26[\mathrm{~dB}]$ higher than that received at 200 [m]; regardless of the carrier frequency and the maximum height. Since such a $26[\mathrm{~dB}]$ difference is more than resolvable by any receiver; it is evident that energy fuses have much higher height resolution than Doppler fuses.

\section{HYBRID FUSE}

Although a Doppler fuse has much less height resolution than an energy fuse; it is more frequency selective. Any signal shifted in frequency by more than the maximum expected Doppler shift will not be received by a Doppler fuse; while it may cause false detonation of an energy fuse. A hybrid fuse detonates when the received signal simultaneously satisfies two conditions: 
a. Its frequency is shifted from that transmitted with the value

$f_{d}=0.02953 f_{[M]} \cdot\left(\sqrt{h_{\max }-10}\right)$.

b. Its power exceeds the critical value $P \cong \frac{P_{T} \cdot \lambda^{2} \cdot \sigma_{0}}{6400 \pi^{2}}=P_{T} \cdot \sigma_{0} \cdot\left(\frac{75}{20 \pi \cdot f_{[M]}}\right)^{2}$.

We shall call a fuse satisfying these conditions a "hybrid fuse". A proposed architecture of such an efficient fuse is introduced in the following section.

\section{A PROPOSED HYBRID FUSE SYSTEM ARCHITECTURE}

It is possible to build a Doppler fuse and an energy fuse, AND their output decisions together and get a hybrid fuse. Another approach will satisfy the same result in a more economic way. Figure (3) shows the functional diagram of this proposed architecture.

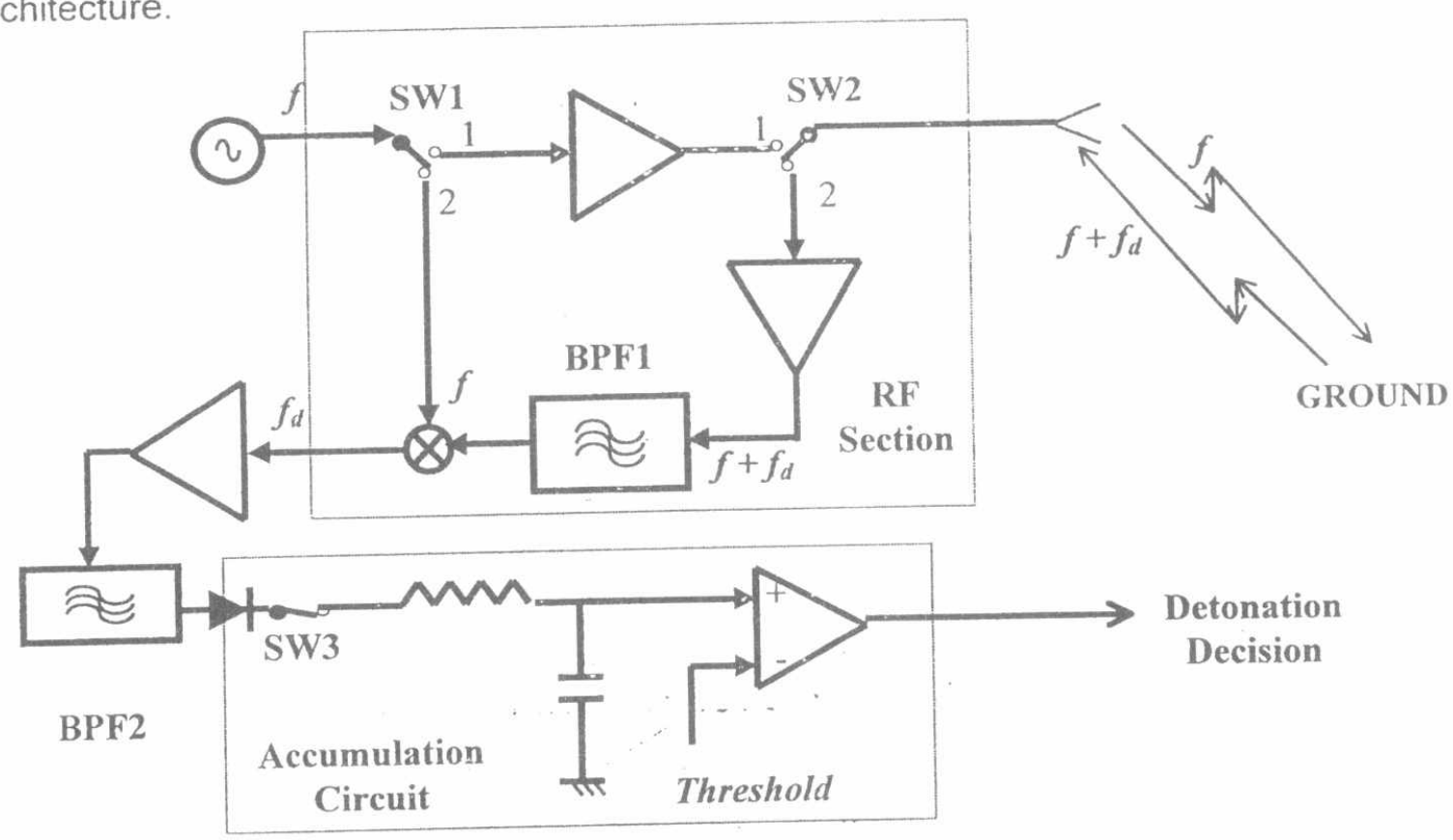

Fig.3 A Proposed Hybrid Fuse Architecture

When the two switches (SW1 and SW2) are in the position 1; the oscillator output is amplified and transmitted. After a certain time $T_{t}$ the position of both switches is reversed and the oscillator output goes to the mixer as a reference coherent source. At the mixer output the Doppler shift is one of the spectral components that can be filtered-out by a band-pass filter (BPF2) tuned to the desired range of relative speeds; which is calculated by the formula (6) for the expected range of maximum heights. Another band-pass filter (BPF1) precedes the mixer to allow only the desired 
frequency band of the reflected signal of interest. The bandwidth of this filter is twice the maximum expected Doppler shift and the central frequency is that of the oscillator. The base band amplifier may precede or follow the BPF2. During every receiving cycle; the detected DC voltage is used to charge a capacitor at a certain time constant. As the output of this accumulating circuit reaches a certain value; corresponding to the power calculated by the formula (14), the detonation decision; based on both Doppler shift and accumulated power, is generated.

\section{EFFECT OF CHOPPING ON THE RECEIVED ENERGY}

To simplify system implementation and minimize its costs ; the proposed fuse uses a single antenna for transmission and reception. There are two main reasons not to use a circulator to handle the transmitted and received signals through that antenna: The first is the size and weight limitations. The other is that its limited isolation (less than $20 \mathrm{~dB}$ ) is much smaller than the difference between the transmitted and the received signal powers which exceeds $58 \mathrm{~dB}$ for the frequencies of interest; according to formula (14). Adopting the transmission-reception switching solution; we have to study the effect of chopping on the received energy and select a suitable value for the switching time rate. Let the transmitted power be :

$$
\begin{aligned}
P_{T}(t) & =P & & \text { for } n \cdot T<t<(n+1) \cdot T \\
& =0 & & \text { for }(n+1) . T<t<(n+2) . T
\end{aligned}
$$

The received power will be:

$$
\begin{aligned}
P_{R}(t) & =P \cdot \sigma_{0} \cdot\left(\frac{75}{2 \pi \cdot h \cdot f_{[M]}}\right)^{2} & & \text { for } n T+(h / 150)<t<(n+1) T+(h / 150)[\mu s] . \\
& =0 & & \text { for }(n+1) T+(h / 150)<t<(n+2) T+(h / 150)[\mu s]
\end{aligned}
$$

We shall study two cases; a fast chopping case where $T=\left(h_{\min } / 150\right)$ and a slow chopping case where $T=\left(h_{\max } / 150\right)$.

\section{First Case: Fast Chopping}

When the propagation time $(h / 150)=2 n T$ no power is received; since the reflected pulse totally arrives during a transmission period. On the other hand, a reflected pulse is totally received when $(h / 150)=(2 n+1) T$. Therefore; the receiving duty cycle is a sawtooth function of the height $h$ with period $2 \mathrm{~T}$ :

$$
\begin{aligned}
\delta_{R} & =((h / 150)-2 n T) / 2 T & & \text { for } 2 n T<<(h / 150)<(2 n+1) T \\
& =1-[(h / 150)-2 n T] / 2 T & & \text { for }(2 n+1) T<(h / 150)<2(n+1) T
\end{aligned}
$$

Multiplying the received power with the maximum duty ratio (0.5) we get the envelope of the received power as a function of height: 
$P_{r} \cdot \delta_{\max }=P_{T} \cdot \sigma_{0} \cdot\left(\frac{75}{2 \pi \cdot h \cdot f_{[M]}}\right)^{2} *(0.5)=P_{T} \cdot \sigma_{0} \cdot\left(\frac{2.984}{h \cdot f_{[M]}}\right)^{2}=8.9\left(\frac{P_{T} \cdot \sigma_{0} .}{\left(h \cdot f_{[M]}\right)^{2}}\right)$

The actually received power will vary between this value and zero as the fuse height decreases. It is shown in Fig.(4) for a 100 [mw] peak transmitted power and 1000 [m] maximum height. Since the received power increases sharply at the smallest heights we have chosen $T=10[\mathrm{~m}] / 150[\mu \mathrm{s} / \mathrm{m}]=67[\mathrm{~ns}]$. Fig. 5 shows the received power, the output and accumulated voltages as functions of time for the same case.

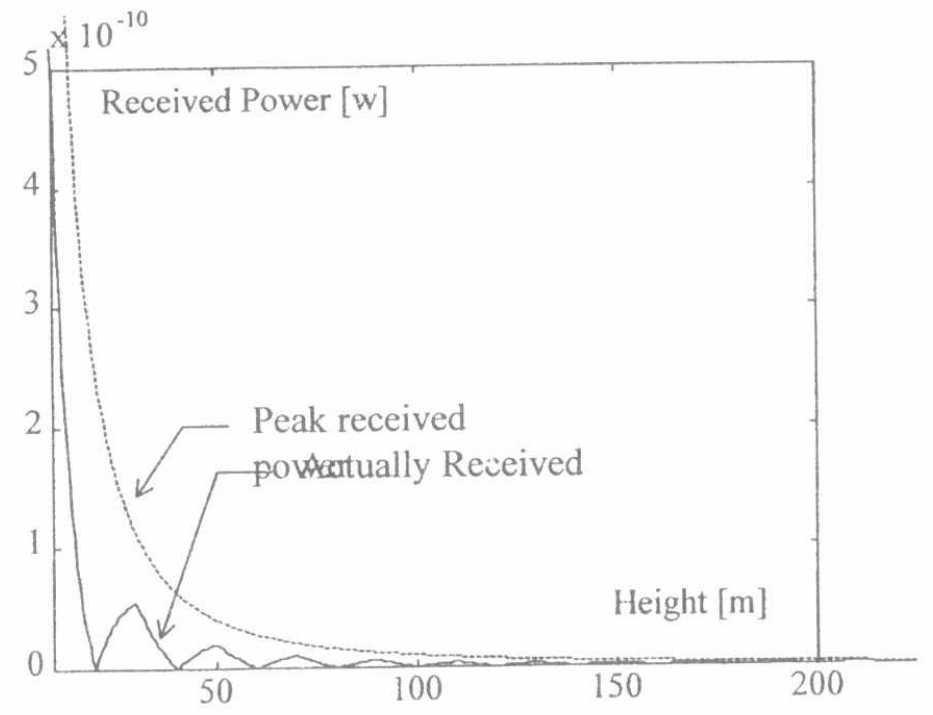

Fig.4 Variation of the received power with fuse height

\section{Second Case: Slow Chopping}

In this case we choose $T=1000[\mathrm{~m}] / 150[\mu \mathrm{s} / \mathrm{m}]=6.7[\mu \mathrm{s}]$. All the power transmitted during a single transmission period $\mathrm{T}$ will be reflected before the next transmission period starts. The reception time per cycle is $(h / 150)[\mu s]$. Multiplying with the received power we get

The received energy per cycle $=P_{T} \sigma_{0}\left(\frac{75}{2 \pi h \cdot f_{[M]}}\right)^{2} \frac{h}{150}=\frac{0.95 P_{T} \sigma_{0}}{h \cdot\left(f_{[M]}\right)^{2}}$

The received energy per unit time is this value divided by $2 T$; i.e.

The received power $=\frac{0.95 P_{T} \cdot \sigma_{0}}{2 * 6.7 h\left(f_{[M]}\right)^{2}}=\frac{0.071 P_{T} \cdot \sigma_{0}}{h \cdot\left(f_{[M]}\right)^{2}} \quad[\mathrm{~W}]$

Comparing (20) with (18) ; we can deduce that the slow chopping case gives higher energy accumulating rates for $h>125[\mathrm{~m}]$ if $h_{\max }$ is $100[\mathrm{~m}]$. For long range projectiles with $10[\mathrm{~km}]$ maximum height the slow chopping case gives higher power for $h>1250[\mathrm{~m}]$ where the reflected power is still very small. Therefore, slow 
chopping is preferred for relatively short range projectiles, while fast chopping is more convenient for long-range projectiles.
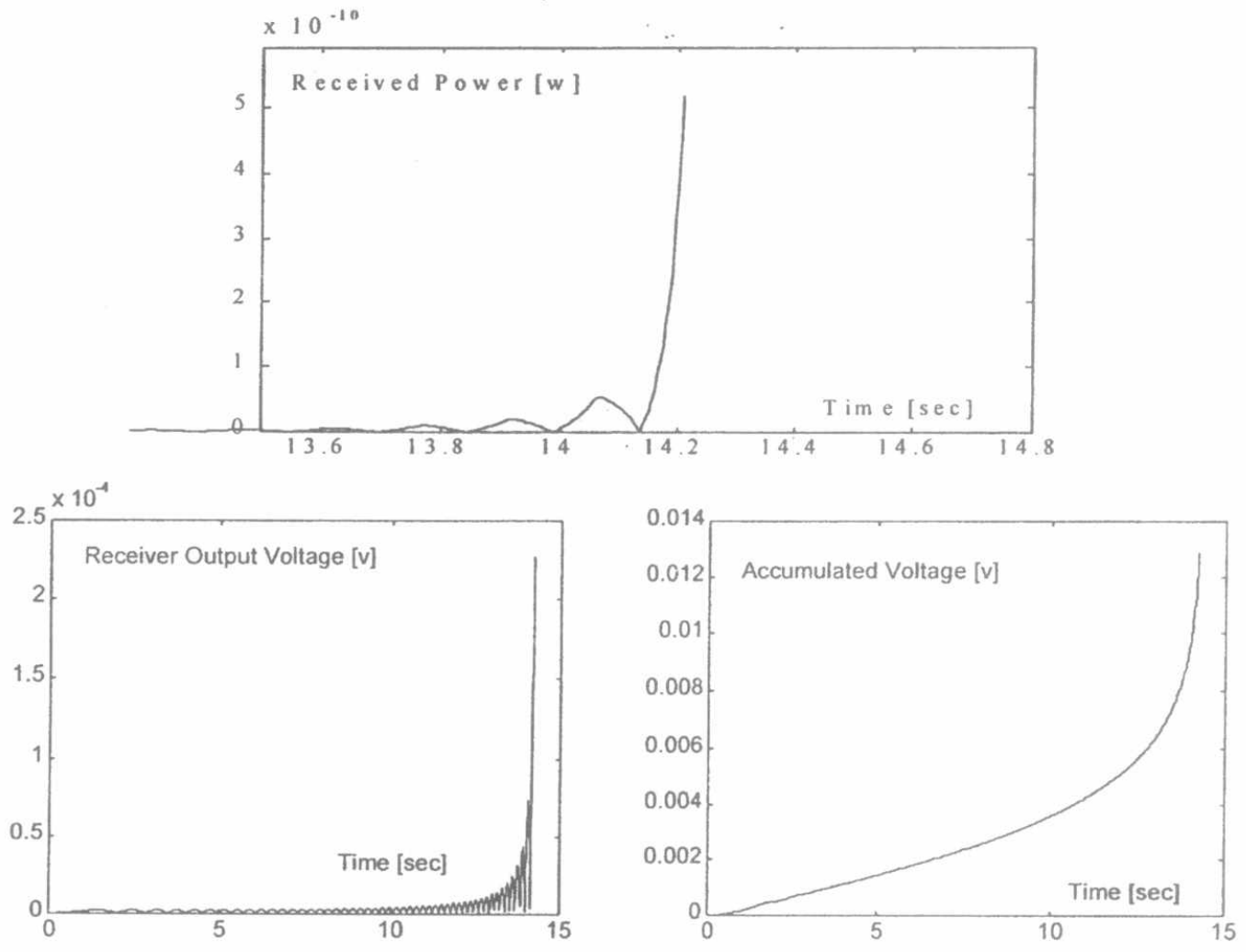

Fig. 5 The received power, the output and accumulated voltages v0s time

\section{CONCLUSION}

1. Energy fuses have higher height resolution than Doppler fuses, while Doppler fuses are more interference immune; being more selective.

2. A hybrid fuse is the optimal approach to a reliable fuse specification.

3. A proposed hybrid fuse design approach has been introduced and explained. This proposed fuse design uses a single antenna chopped between transmission and reception. After an analytical study of two options; slow chopping at 6.7 [ $\mu$ s] rate has been selected and the accumulated energy has been calculated for a short-range projectile. Fast chopping is preferred for long-range projectiles.

\section{REFERENCE}

1. I. H. Shames, Engineering Mechanics: Statics and Dynamics, Prentice Hall.1971 
